\title{
Determinants and consequences of insulin initiation for type 2 diabetes in France: analysis of the National Health and Wellness Survey
}

This article was published in the following Dove Press journal:

Patient Preference and Adherence

7 October 2013

Number of times this article has been viewed

\author{
Gérard Reach' \\ Véronique Le Pautremat ${ }^{2}$ \\ Shaloo Gupta ${ }^{3}$ \\ 'Department of Endocrinology, \\ Diabetes, and Metabolic Diseases, \\ Avicenne Hospital APHP, and EA \\ 34I2, CRNH-IdF, Paris I 3 University, \\ Sorbonnne Paris Cité, Bobigny, France; \\ ${ }^{2}$ Kantar Health, Paris, France; ${ }^{3}$ Kantar \\ Health, Princeton, NJ, USA
}

Background: The aim of the study was to identify the intrinsic patient characteristics and extrinsic environmental factors predicting prescription and use and, more specifically, early initiation (up to 5 years of disease duration) of insulin for type 2 diabetes in France. A secondary objective was to evaluate the impact of insulin therapy on mental and physical quality of life and patient adherence.

Methods: The data used in this study were derived from the 2008, 2010, and 2011 France National Health and Wellness Survey. This survey is an annual, cross-sectional, self-administered, Internetbased questionnaire among a nationwide representative sample of adults (aged 18 years or older). Of the total of 45,958 persons recruited in France, 1,933 respondents (deduped) were identified as diagnosed with type 2 diabetes. All unique respondents from the three waves, currently using insulin or oral bitherapy or tritherapy at the time of assessment, were included in this analysis.

Results: Early (versus late) initiation of insulin therapy was 9.9 times more likely to be prescribed by an endocrinologist or diabetologist than by a primary care physician $(P<0.0001)$. Younger age at diagnosis and current smoking habits were significant predictors of early (versus late) insulin initiation (odds ratio [OR] 1.031, 95\% confidence interval [CI] 1.005-1.059, $P=0.0196$, and OR $2.537,95 \%$ CI $1.165-5.524, P=0.0191$, respectively). Patients with a yearly income $\geq € 50,000$ were less likely to be put on insulin early $(P=0.0399)$. A link between insulin prescription and complications was shown only in univariate analysis. Mental quality of life was lower in patients on early (versus late) insulin, but only in patients with diabetes-related complications. Insulin users (versus oral bitherapy or tritherapy users) had 3.0 times greater odds of being adherent than uncontrolled oral bitherapy or tritherapy users (OR 2.983, 95\% CI 1.37-6.495, $P=0.0059$ ).

Conclusion: This study confirms the role of specialists in early initiation of insulin, and the data presented herein reflect the fact that early initiation is more frequent in younger patients, patients with diabetes-related complications, and current smokers, and less frequent in patients with a higher income. Moreover, we observed that being treated with insulin was not associated with deterioration in quality of life, and insulin-treated patients were more often adherent than uncontrolled oral bitherapy or tritherapy users. These data suggest that doctors' concerns about patient adherence and detrimental effects on quality of life should not be a barrier to their decision regarding early initiation of insulin therapy. Due to the nature of this cross-sectional survey (eg, inability to assess treatment flow), further research is needed to confirm its findings.

Keywords: type 2 diabetes, early insulin initiation, quality of life, adherence, psychological insulin resistance, clinical inertia

\section{Introduction}

The prevalence of type 2 diabetes is increasing worldwide, with the number of affected individuals expected to double by $2050 .{ }^{1}$ Diabetes is a leading cause of kidney failure, 
blindness, leg amputation, and myocardial infarction. There is evidence that early control of blood glucose can help prevent these diabetes-related complications. ${ }^{2}$ Current treatment is based on a stepwise approach starting with changes in lifestyle and progressively introducing oral antidiabetic agents, with the aim of maintaining glycated hemoglobin $\left(\mathrm{HbA}_{1 \mathrm{c}}\right)$ levels below a defined target. This target is defined according to the patient's characteristics. ${ }^{3-5}$ In the current paradigm, insulin is usually considered to be the last step in treatment intensification.

Prescribing insulin at later stages of type 2 diabetes disease progression has recently been challenged, arguing that a delay in insulin initiation may affect the patient's long-term prognosis. Indeed, it is well known that elevated fasting plasma glucose levels are primarily due to an increase in hepatic glucose production, secondary to an insufficient endogenous insulin secretion needed to overcome insulin resistance. ${ }^{6}$ Furthermore, there is a well documented decline in insulin secretion due to beta cell exhaustion, and it has been suggested that early use of insulin may suppress inflammation and glucolipotoxicity, which results in autoaggravation of the disease. ${ }^{7}$

However, early introduction of insulin may represent a challenge with regard to the well known "psychological insulin resistance" status affecting both patients and doctors. This results in delayed insulin prescription when it would be appropriate according to current guidelines. Psychological insulin resistance, ${ }^{8}$ which represents a typical case of clinical inertia, ${ }^{9-14}$ may be due in part, on the side of the doctor, to the supposed effect of insulin treatment on patients' quality of life which may hinder future adherence. ${ }^{15-19}$ On the other hand, nonadherence to long-term therapies also represents a barrier to the efficiency of care, ${ }^{20-22}$ even if it seems that doctors' clinical inertia is actually more frequent than patient nonadherence. ${ }^{23}$

In this context, the objective of this study using data from the National Health and Wellness Survey (NHWS) carried out in France was to identify the intrinsic and extrinsic determinants of insulin prescription, and more specifically of early insulin initiation (being defined as 5 years or less following diagnosis) in type 2 diabetes, and to evaluate the impact of insulin therapy on mental and physical quality of life and patient adherence.

\section{Materials and methods National Health and Wellness Survey sample}

The study sample and data were taken from the 2008, 2010, and 2011 waves $(2008, \mathrm{n}=15,457 ; 2010, \mathrm{n}=15,501 ; 2011$, $\mathrm{n}=15,000)$ of the French NHWS. The NHWS is an annual Internet-based questionnaire developed by Kantar Health and the Ailment Panel of Lightspeed Research. It is a crosssectional study of subjects aged 18 years or older, conducted with a strictly identical methodology for the 3 years (2008, 2010 , and 2011). Only a small proportion of individuals from the sample were common between waves (approximately one in five) and data from recent participation were retained.

The primary objective of the NHWS is to provide a comprehensive database of epidemiological and treatment information, health care attitudes, behaviors, demographic and disease characteristics, and health-related outcomes. The 2011, 2010, and 2008 surveys employ a stratified random sample (with both sex and age group quotas), in order to replicate the demographic composition of each of the population of each individual country. Representation of NHWS data has been validated against reliable sources, including government agencies' health statistics and nonaffiliated third parties. Results are projected to reflect the total population in each country using known population characteristics. In France, data are weighted by sex and age using the United States Bureau of the Census and Organization for Economic Cooperation and Development.

A self-administered questionnaire is completed by a sample population identified through a web-based consumer panel. All data from the NHWS are self-reported by participating respondents. All respondents received and agreed with the informed consent form provided, and the study was approved by the Essex Institutional Review Board (Lebanon, NJ, USA). Of the total 45,958 persons recruited (three waves deduped study sample), 1,933 respondents were identified as reporting a physician diagnosis of type 2 diabetes, comprising 591 from the 2008 wave, 649 from 2010, and 693 from 2011 (respectively $3.8 \%, 4.2 \%$, and $4.3 \%$ of the general population of adults). All unique respondents diagnosed with type 2 diabetes currently using insulin or oral bitherapy or tritherapy at the time of assessment were included ( $n=713$, see Table 1$)$; this choice was justified by the fact that, both according to current guidelines and as a result of doctors' and patients' psychological insulin resistance, patients treated with dual therapy or tritherapy have a greater likelihood of being switched to insulin than those treated with monotherapy.

\section{Measures and survey instruments Independent variables}

We first compared all patients on bitherapy or tritherapy $(\mathrm{n}=443)$ and all insulin users $(\mathrm{n}=270)$. Second, we compared early and late initiation of insulin: based on calcula- 
Table I Sample sizes

\begin{tabular}{ll}
\hline Groups (deduped)* & $\begin{array}{l}\text { Sample } \\
\text { size (n) }\end{array}$ \\
\hline All oral bitherapy or tritherapy users & 443 \\
Uncontrolled** bitherapy or tritherapy users & 105 \\
without any complications & 360 \\
All insulin users & 270 \\
With: & 143 \\
Early insulin initiation (5 years or less) & 77 \\
Early insulin initiation (5 years or less) without any & \\
complication^ & 141 \\
Short duration of insulin (5 years or less) & 94 \\
Short duration of insulin (5 years or less) without any & \\
complication^ &
\end{tabular}

Notes: *Users of glucagon-like peptide-I were excluded from this analysis due to their small sample size $(n=46)$; **uncontrolled users were defined as having an $\mathrm{HbA}_{\mathrm{lc}}>7 \%$ or, if they were missing their $\mathrm{HbA}_{\mathrm{lc}}$ level their fasting plasma glucose was $>130 \mathrm{mg} / \mathrm{dL}$; $\wedge$ a complication was defined as reporting having myocardial infarction, stroke, transient ischemic attack, diabetic retinopathy, diabetic peripheral neuropathy, kidney damage, end organ damage (only collected in 2010 and 20lI), or foot ulcer.

tion of the number of years between diagnosis of type 2 diabetes and initiation of insulin, with early insulin defined as 5 years or less $(n=143)$. The control group $(n=124)$ consisted of those patients who were prescribed insulin later. Third, another independent variable of interest was the duration of insulin use, using a median split of the number of years using insulin, ie, 5 years or less versus 6 years or more (Table 1).

\section{Covariates}

Regardless of how early initiation is defined, great care must be taken in isolating the effect of early initiation or beginning insulin on health outcomes, given the crosssectional, observational nature of the NHWS. Insulin can be initiated for a variety of reasons as physicians attempt to manage risk in their patients. Irrespective of outcomes, the model included the following predictors: age/age at diagnosis, duration of type 2 diabetes, sex, education, household income, and employment type (see Table 2). The following health information was also included: body mass index, smoking status, alcohol use, exercise habits, diabetes complications experienced, prescribing physician, being afraid of needles, $\mathrm{HbA}_{1 \mathrm{c}}$, fasting glucose, treatment satisfaction, and Charlson comorbidity index. The Charlson comorbidity index is calculated by weighting the presence of specific comorbidities based on their association with future mortality and summing the results. Models consisting of respondents with uncontrolled type 2 diabetes did not include $\mathrm{HbA}_{1 \mathrm{c}}$ level and fasting glucose as predictors.
Table 2 Definition of covariates

\begin{tabular}{|c|c|}
\hline Parameter & Reference \\
\hline Age/age at diagnosis & - \\
\hline \multicolumn{2}{|l|}{ Duration of type 2 diabetes } \\
\hline Sex & Male \\
\hline College degree + & Less than college degree \\
\hline \multicolumn{2}{|l|}{ Income } \\
\hline$€ 20,000$ to $<€ 50,000$ & $<€ 20,000$ \\
\hline \multicolumn{2}{|l|}{$\geq € 50,000$} \\
\hline \multicolumn{2}{|l|}{ Decline to answer } \\
\hline Employed full-time/part-time/ & Unemployed \\
\hline \multicolumn{2}{|l|}{ self-employed } \\
\hline Currently drinking alcohol & No current alcohol use \\
\hline Currently smoking & Not a current smoker \\
\hline Currently exercising & No current exercise \\
\hline \multicolumn{2}{|l|}{ Body mass index } \\
\hline Overweight & Normal/underweight \\
\hline \multicolumn{2}{|l|}{ Obese } \\
\hline \multicolumn{2}{|l|}{ Declined } \\
\hline Charlson comorbidity index & - \\
\hline \multicolumn{2}{|l|}{ Complications experienced } \\
\hline Macular edema or diabetic retinopathy & Not experienced \\
\hline \multicolumn{2}{|l|}{ Neuropathic pain } \\
\hline \multicolumn{2}{|l|}{ Kidney disease } \\
\hline \multicolumn{2}{|l|}{ Foot or leg ulcer } \\
\hline Prescribing physician, & Specialist \\
\hline \multicolumn{2}{|l|}{ general practitioner } \\
\hline Strongly agree/agree with being & Neutral/disagree/strongly \\
\hline \multirow[t]{2}{*}{ afraid of needles } & disagree with being afraid \\
\hline & of needles \\
\hline $\mathrm{HbA}_{\mathrm{Ic}}$ & $\leq 7 \%$ \\
\hline \multicolumn{2}{|l|}{$>7 \%$} \\
\hline \multicolumn{2}{|l|}{ Unknown } \\
\hline \multicolumn{2}{|l|}{ Fasting glucose } \\
\hline$\geq 130 \mathrm{mg} / \mathrm{dL}$ & $\leq 130 \mathrm{mg} / \mathrm{dL}$ \\
\hline \multicolumn{2}{|l|}{ Unknown } \\
\hline Very/extremely satisfied with & Less satisfaction with \\
\hline diabetes treatment & diabetes treatment \\
\hline
\end{tabular}

Note: Models representing uncontrolled individuals did not include $\mathrm{HbA}_{\mathrm{lc}}$ levels or fasting glucose as covariates.

\section{Quality of life and medication adherence outcomes}

The following measures of quality of life (validated scales) were used in this analysis: physical (PCS) and mental (MCS) component summary scores from the Short Form Survey Instrument Version 2 (SF-12v2). The SF-12v2 is a multipurpose generic measure of health status, consisting of 12 questions designed to assess physical functioning, role limitations due to physical health problems, body pain, general health, vitality, social functioning, role limitations due to emotional problems, and mental health. Scores for the PCS and MCS rely on norm-based scoring, with higher scores indicating better quality of life. The average score is $50 .{ }^{24}$ 
Medication adherence was assessed using the Morisky Medication Adherence Scale (MMAS). The MMAS consists of four yes/no questions that assess the general adherence of using prescribed medication. ${ }^{25}$ The total score varies from 0 to 4, with lower scores indicating greater adherence; adherence $(0)$ versus nonadherence (1-4) were compared. Medication adherence data was only collected in 2010 and 2011. Therefore, the sample size is smaller compared with the other metrics.

\section{Statistical analyses}

Bivariate and multivariate analyses were conducted. Bivariate analyses were used to compare data between patients on insulin versus patients on oral bitherapy or tritherapy, and data between patients starting early insulin versus late insulin. Chi-square tests were used for categorical variables and the $t$-test was used for continuous variables to determine differences between groups.

The independent variables were used in multivariable analyses to identify differences between two groups on quality of life outcome measures and adherence after adjusting for differences in demographics and patient characteristics. This was achieved by regression modeling (logistic and multivariable linear regressions). For all statistical tests, the applied comparison-wise significance level was a $P$ value $<0.05$.

\section{Results}

\section{Determinants of insulin prescription}

Patients on insulin versus patients on oral bitherapy or tritherapy

The average age of patients on insulin $(n=270)$ and on oral bitherapy or tritherapy $(n=443)$ was comparable (59.14 years versus 60.27 years) while $61.48 \%$ and $69.98 \%$, respectively, were men $(P=0.021)$. Those on insulin were younger at diagnosis (mean 44.6 years versus 49.5 years, $P<0.0001)$, and the average duration of type 2 diabetes since diagnosis was longer (14.7 years versus 10.75 years, $P<0.0001)$. Socioeconomically, they more likely to have less income (<€20,000/year: $32.59 \%$ versus $21.90 \%$, $P=0.0021)$, and were less likely to have a higher level of secondary education (24.44\% versus $33.41 \%, P=0.0096)$. In terms of clinical characteristics, they were less frequently overweight (31.85\% versus $39.95 \%, P=0.0277$ ), had a higher Charlson comorbidity index ( 0.6 versus 0.33 , $P=0.0017)$, significantly more likelihood of myocardial infarction $(P=0.0422)$, and presented more microvascular and macrovascular complications $(P<0.0001)$. They were more often poorly controlled $\left(\mathrm{HbA}_{1 \mathrm{c}}>7 \%\right.$ : $32.96 \%$ versus $17.61 \%, P<0.0001)$. They were more frequently followed up by a prescribing physician who was not a general practitioner $(77.78 \%$ versus $24.15 \%, P<0.0001)$ and were more often aware of their $\mathrm{HbA}_{1 \mathrm{c}}$ level $(P<0.0001)$. Finally, they were significantly more adherent (adherence MMAS $=0: 82.61 \%$ versus $72.36 \%, P=0.0066)$. We did not observe differences in terms of healthy lifestyle (physical exercise), risk factors (alcohol, smoking), or fear about needles (Table 3).

As shown in Table 4, a logistic regression was run to assess factors that influence insulin use versus oral bitherapy or tritherapy use. The probability of taking insulin was raised if the patient presented the following factors, in decreasing order: retinopathy or macular edema (odds ratio [OR] 3.035, 95\% confidence interval $[\mathrm{CI}] 1.317-6.992, P=0.0091)$ and neuropathic pain (OR 2.095, 95\% CI 1.149-3.822, $P=0.0159)$. On the contrary, patients treated by a specialist had a 12 times greater odds of using insulin (OR 0.083 , 95\% CI 0.054-0.128, $P<0.0001)$ versus patients followed up by a prescribing general practitioner, and those who had an income $\geq € 50,000$ per annum were less likely to receive insulin (OR 0.26, 95\% CI 0.119-0.567, $P=0.0007$ ). Finally, older age at diagnosis was associated with less likelihood of being put on insulin (OR 0.974, 95\% CI 0.955-0.994, $P=0.0117)$. Overweight and unknown fasting glucose were marginally significant predictors of oral use.

\section{Factors influencing early initiation of insulin}

As shown in Table 5, patients were younger in the type 2 diabetes early insulin initiation group $(n=143)$ than in the type 2 diabetes initiated later with insulin group $(n=124)$, ie, 56.37 years versus 62.6 years $(P<0.0001)$ and this was confirmed in the younger age group at initiation of insulin (47.42 years versus 56.86 years, respectively, $P<0.0001$ ). Fewer males initiated insulin early compared with the late insulin initiation group (55.24\% versus $68.55 \%$ respectively, $P<0.025)$. Socioeconomically, they had a lower income ( $<€ 20,000$ per year: $38.46 \%$ versus $26.61 \%, P=0.0384$ ). In terms of lifestyle, they were more likely to smoke $(23.08 \%$ versus $10.48 \%, P=0.0053$ ), and reported less controlled type 2 diabetes $\left(\mathrm{HbA}_{1 \mathrm{c}}>7 \%, 25.87 \%\right.$ versus $41.94 \%$, $P<0.0057$ ).

As shown in Table 6, a logistic regression was run to assess factors that influence early initiation of insulin ( $\leq 5$ years) versus late initiation using a median split (Table 5). Younger age at diagnosis and currently smoking were significant predictors of early insulin initiation (OR 1.031, 95\% CI $1.005-1.059, P=0.0196$, and OR 2.537, 95\% CI 1.165-5.524, 
Table 3 Analyzed population: all insulin users versus all users of bitherapy or tritherapy

\begin{tabular}{|c|c|c|c|c|c|}
\hline & \multicolumn{2}{|c|}{$\begin{array}{l}\text { All insulin users } \\
n=\mathbf{2 7 0}\end{array}$} & \multicolumn{2}{|c|}{$\begin{array}{l}\text { All bi- or tritherapy } \\
\text { users } \\
n=443\end{array}$} & \multirow[t]{2}{*}{$P$-value } \\
\hline & $\mathbf{n}$ & $\%$ & $\mathbf{n}$ & $\%$ & \\
\hline Age (mean, SD) & 59.14 & 10.86 & 60.27 & 9.32 & 0.1554 \\
\hline Age at diagnosis & 44.6 & 11.95 & 49.53 & 10.39 & $<0.0001$ \\
\hline Age at insulin initiation & 51.61 & 13.03 & - & - & N/A \\
\hline Years diagnosed & 14.66 & 9.88 & 10.75 & 7.61 & $<0.0001$ \\
\hline \multicolumn{6}{|l|}{ Sex } \\
\hline Male & 166 & $61.48 \%$ & 310 & $69.98 \%$ & 0.0213 \\
\hline Female & 104 & $38.52 \%$ & 133 & $30.02 \%$ & 0.0213 \\
\hline Currently employed & 76 & $28.15 \%$ & 121 & $27.31 \%$ & 0.8096 \\
\hline \multicolumn{6}{|l|}{ Household income } \\
\hline$<20,000 €$ & 88 & $32.59 \%$ & 97 & $21.90 \%$ & 0.0021 \\
\hline $20,000 €$ to $<50,000 €$ & 138 & $51.11 \%$ & 233 & $52.60 \%$ & 0.7006 \\
\hline $50,000 €$ or more & 22 & $8.15 \%$ & 65 & $14.67 \%$ & 0.0061 \\
\hline Decline to answer & 22 & $8.15 \%$ & 48 & $10.84 \%$ & $0.228 I$ \\
\hline College education & 66 & $24.44 \%$ & 148 & $33.41 \%$ & 0.0096 \\
\hline \multicolumn{6}{|l|}{$\mathrm{BMI}$} \\
\hline Underweight & 0 & $0.00 \%$ & I & $0.23 \%$ & $\mathrm{~N} / \mathrm{A}$ \\
\hline Normal weight & 40 & $14.81 \%$ & 45 & $10.16 \%$ & 0.0735 \\
\hline Overweight & 86 & $31.85 \%$ & 177 & $39.95 \%$ & 0.0277 \\
\hline Obese & 135 & $50.00 \%$ & 209 & $47.18 \%$ & 0.4651 \\
\hline Decline to answer & 9 & $3.33 \%$ & 11 & $2.48 \%$ & 0.5197 \\
\hline \multicolumn{6}{|l|}{ Health behaviors } \\
\hline Currently drink & 190 & $70.37 \%$ & 336 & $75.85 \%$ & 0.1126 \\
\hline Currently smoke & 47 & $17.41 \%$ & 75 & $16.93 \%$ & 0.8701 \\
\hline Regularly exercise & 141 & $52.22 \%$ & 238 & $53.72 \%$ & 0.697 \\
\hline Charlson comorbidity index (mean, SD) & 0.6 & 1.33 & 0.33 & 0.69 & 0.0017 \\
\hline \multicolumn{6}{|l|}{ Comorbidities } \\
\hline Depression & 36 & $13.33 \%$ & 50 & $11.29 \%$ & 0.4242 \\
\hline Myocardial infarction & 23 & $8.52 \%$ & 20 & $4.51 \%$ & 0.0422 \\
\hline Stroke & 12 & $4.44 \%$ & 10 & $2.26 \%$ & 0.1294 \\
\hline TIA & 4 & $1.48 \%$ & 2 & $0.45 \%$ & 0.1995 \\
\hline Congestive heart failure & 8 & $2.96 \%$ & 7 & $1.58 \%$ & 0.246 \\
\hline Hypertension & 111 & $41.11 \%$ & 205 & $46.28 \%$ & 0.1771 \\
\hline Angina & 27 & $10.00 \%$ & 50 & $11.29 \%$ & 0.5869 \\
\hline Arrhythmia & 13 & $4.81 \%$ & 31 & $7.00 \%$ & 0.2208 \\
\hline \multicolumn{6}{|l|}{ Prescribing physician } \\
\hline PCP & 53 & $19.63 \%$ & 334 & $75.40 \%$ & $<0.0001$ \\
\hline Endocrinologist/diabetologist & 210 & $77.78 \%$ & 107 & $24.15 \%$ & $<0.000$ I \\
\hline Nurse practitioner/physician assistant & 0 & $0.00 \%$ & 0 & $0.00 \%$ & N/A \\
\hline Other & 7 & $2.59 \%$ & 2 & $0.45 \%$ & 0.0361 \\
\hline Agree/strongly agree to being afraid of needles & 24 & $8.89 \%$ & 47 & $10.61 \%$ & 0.4485 \\
\hline \multicolumn{6}{|l|}{ Microvascular complications } \\
\hline Macular edema & 43 & $15.93 \%$ & 14 & $3.16 \%$ & $<0.0001$ \\
\hline Neuropathic pain & 53 & $19.63 \%$ & 33 & $7.45 \%$ & $<0.0001$ \\
\hline Kidney damage & 28 & $10.37 \%$ & 11 & $2.48 \%$ & $<0.0001$ \\
\hline End organ damage $(2010,2011$ only) & 14 & $7.61 \%$ & 5 & $1.55 \%$ & 0.0038 \\
\hline Foot ulcer & 14 & $5.19 \%$ & 16 & $3.61 \%$ & 0.3305 \\
\hline At least one microvascular complication & 104 & $38.52 \%$ & 64 & $14.45 \%$ & $<0.000$ I \\
\hline At least one complication (TIA, Stroke, & 119 & $44.07 \%$ & 83 & $18.74 \%$ & $<0.0001$ \\
\hline \multicolumn{6}{|l|}{ HA or microvascular complication) } \\
\hline \multicolumn{6}{|l|}{$\mathrm{HbA}_{\mathrm{Ic}}(\%)$} \\
\hline $\mathrm{HbA}_{\mathrm{Ic}}>7$ & 89 & $32.96 \%$ & 78 & $17.61 \%$ & $<0.0001$ \\
\hline $\mathrm{HbA}_{\mathrm{Ic}}$ (missing) & 94 & $34.81 \%$ & 228 & $51.47 \%$ & $<0.000$ I \\
\hline
\end{tabular}


Table 3 (Continued)

\begin{tabular}{|c|c|c|c|c|c|}
\hline & \multicolumn{2}{|c|}{$\begin{array}{l}\text { All insulin users } \\
n=\mathbf{2 7 0}\end{array}$} & \multicolumn{2}{|c|}{$\begin{array}{l}\text { All bi- or tritherapy } \\
\text { users } \\
n=443\end{array}$} & \multirow[t]{2}{*}{$P$-value } \\
\hline & n & $\%$ & $\mathbf{n}$ & $\%$ & \\
\hline \multicolumn{6}{|l|}{ Fasting glucose (mg/dl) } \\
\hline Fasting glucose $>130$ & 42 & $15.56 \%$ & 45 & $10.16 \%$ & 0.0409 \\
\hline Fasting glucose (missing) & 152 & $56.30 \%$ & 311 & $70.20 \%$ & 0.0002 \\
\hline \multicolumn{6}{|l|}{ Satisfaction with treatment } \\
\hline Very/extremely satisfied with treatment & 149 & $55.60 \%$ & 227 & $51.24 \%$ & 0.2592 \\
\hline Very/extremely dissatisfied with treatment & 40 & $14.93 \%$ & 51 & $11.51 \%$ & 0.1991 \\
\hline \multicolumn{6}{|l|}{ Morisky adherence* $(2010,201$ I only) } \\
\hline Compliant $(\mathrm{MMAS}=0)$ & 152 & $82.61 \%$ & 233 & $72.36 \%$ & 0.0066 \\
\hline Forget to take medication & 25 & $13.59 \%$ & 80 & $24.84 \%$ & 0.0014 \\
\hline Careless about medication & 12 & $6.52 \%$ & 36 & $11.18 \%$ & 0.0667 \\
\hline Stop when feeling better & 1 & $0.54 \%$ & 5 & $1.55 \%$ & 0.2508 \\
\hline Stop when feeling worse & 5 & $2.72 \%$ & 16 & $4.97 \%$ & 0.1876 \\
\hline
\end{tabular}

Notes: *Assessed using the MMAS. The MMAS includes four items ("do you ever forget to take your medicine?"; "are you careless at times about taking your medicine?"; "when you feel better do you sometimes stop taking your medicine?"; and "sometimes if you feel worse when you take your medicine, do you stop taking it?"). All items have a dichotomous yes/no response scale and are summed to form a total score (which varies from 0 to 4, with lower scores indicating greater adherence).

Abbreviations: BMI, body mass index; MMAS, Morisky Medication Adherence Scale; SD, standard deviation; HA, heart attack; TIA, transient ischemic attack; PCP, primary care physician.

$P=0.0191$, respectively). Inversely, high income (€20,000$€ 50,000)$ was a significant predictor of late insulin initiation (OR 0.452, 95\% CI 0.239-0.856, $P=0.0148$ ). It should be noted that a currently uncontrolled $\mathrm{HbA}_{1 \mathrm{c}}$ level was a marginally significant predictor of late insulin initiation (OR 0.549, 95\% CI 0.28-1.076, $P=0.0807$ ).

A logistic regression was also run to assess the factors that influence early insulin initiation versus the subgroup of uncontrolled oral bitherapy or tritherapy users. From Table 6, we can see that high income was also associated with late insulin initiation, with patients receiving an income of $\geq € 50,000$ per annum having lower odds of being put on insulin early (OR 0.26, 95\% CI 0.072-0.94, $P=0.0399$ ). It should be noted that the odds of early initiation of insulin therapy prescribed by an endocrinologist or diabetologist were 9.9 greater compared with a primary care physician (OR 0.101, 95\% CI 0.05-0.204, $P<0.0001$ ).

\section{Predictors of control of type 2 diabetes}

As shown in Table 7, when comparing controlled patients (all therapies, $\mathrm{n}=224)$ versus uncontrolled $\left(\mathrm{HbA}_{1 \mathrm{c}}<7 \%\right)$ patients $(n=208)$, those with controlled diabetes were older at diagnosis (48.16 years versus 45.38 years, $P=0.0109$ ), were less often treated with insulin (38.84\% versus $49.52 \%$, $P=0.0254)$, had macular edema less often (6.25\% versus $14.42 \%, P=0.0055)$, or at least one microvascular complication $(20.09 \%$ versus $33.65 \%, P=0.0015)$, were very satisfied with treatment more often $(65.18 \%$ versus $46.63 \%$, $P<0.0001$ ), and forgot less often to take their medication (15.87\% versus $25.00 \%, P=0.0411$ ).

A logistic regression was run to assess the factors that influence control of $\mathrm{HbA}_{1 \mathrm{c}}$ (all types of treatment, Table 8). Three factors were associated with controlled diabetes, ie, patient satisfaction with treatment (OR 2.545, 95\% CI $1.556-4.16, P=0.0002)$, a short duration of diabetes (OR

Table 4 Statistically significant factors influencing prescription of insulin: insulin users versus bitherapy or tritherapy users $(n=705)$

\begin{tabular}{|c|c|c|c|c|c|c|c|}
\hline Parameter & Estimate & OR & 95\% LCL for OR & $95 \%$ UCL for OR & SE & Chi-square & $P$-value \\
\hline Age at diagnosis & -0.026 & 0.974 & 0.955 & 0.994 & 0.0103 & 6.3489 & 0.0117 \\
\hline Income: $\geq € 50,000$ & -1.349 & 0.26 & 0.119 & 0.567 & 0.3987 & 11.439 & 0.0007 \\
\hline Income: declined to answer & -0.831 & 0.436 & 0.201 & 0.944 & 0.3949 & 4.4294 & 0.0353 \\
\hline $\begin{array}{l}\text { Macular edema or diabetic } \\
\text { retinopathy }\end{array}$ & 1.1102 & 3.035 & 1.317 & 6.992 & 0.4258 & 6.7983 & 0.0091 \\
\hline Neuropathic pain & 0.7397 & 2.095 & 1.149 & 3.822 & 0.3067 & 5.8187 & 0.0159 \\
\hline Prescribing physician: GP & -2.486 & 0.083 & 0.054 & 0.128 & 0.2201 & 127.62 & $<0.0001$ \\
\hline
\end{tabular}

Abbreviations: GP, general practitioner; OR, odds ratio; SE, standard error; LCL, lower confidence limit; UCL, upper confidence limit. 
Table 5 Analyzed population: early versus late insulin initiation

\begin{tabular}{|c|c|c|c|c|c|}
\hline & \multicolumn{2}{|c|}{$\begin{array}{l}\text { Early insulin initiation } \\
(5 \text { years or less) } \\
n=\mid 43\end{array}$} & \multicolumn{2}{|c|}{$\begin{array}{l}\text { Late insulin initiation } \\
\text { ( } 6 \text { years or more) } \\
n=124\end{array}$} & \multirow[t]{2}{*}{$P$-value } \\
\hline & $\mathbf{n}$ & $\%$ & n & $\%$ & \\
\hline Age (mean, SD) & 56.37 & 11.09 & 62.6 & 8.81 & $<0.000$ I \\
\hline Age at diagnosis & 45.74 & 13.52 & 43.29 & 9.73 & 0.0875 \\
\hline Age at insulin initiation & 47.42 & 13.95 & 56.86 & 9.41 & $<0.0001$ \\
\hline \multicolumn{6}{|l|}{ Sex } \\
\hline Male & 79 & $55.24 \%$ & 85 & $68.55 \%$ & 0.025 \\
\hline Female & 64 & $44.76 \%$ & 39 & $31.45 \%$ & 0.025 \\
\hline Currently employed & 44 & $30.77 \%$ & 30 & $24.19 \%$ & 0.2295 \\
\hline \multicolumn{6}{|l|}{ Household income } \\
\hline$<20,000 €$ & 55 & $38.46 \%$ & 33 & $26.61 \%$ & 0.0384 \\
\hline $20,000 €$ to $<50,000 €$ & 62 & $43.36 \%$ & 73 & $58.87 \%$ & 0.0112 \\
\hline $50,000 €$ or more & 11 & $7.69 \%$ & 11 & $8.87 \%$ & 0.7288 \\
\hline Decline to answer & 15 & $10.49 \%$ & 7 & $5.65 \%$ & 0.1435 \\
\hline College education & 37 & $25.87 \%$ & 28 & $22.58 \%$ & 0.5314 \\
\hline \multicolumn{6}{|l|}{ BMI } \\
\hline Underweight & 0 & $0.00 \%$ & 0 & $0.00 \%$ & $\mathrm{~N} / \mathrm{A}$ \\
\hline Normal weight & 22 & $15.38 \%$ & 15 & $12.10 \%$ & 0.4358 \\
\hline Overweight & 41 & $28.67 \%$ & 45 & $36.29 \%$ & 0.1864 \\
\hline Obese & 74 & $51.75 \%$ & 61 & $49.19 \%$ & 0.6779 \\
\hline Decline to answer & 6 & $4.20 \%$ & 3 & $2.42 \%$ & 0.4149 \\
\hline \multicolumn{6}{|l|}{ Health behaviors } \\
\hline Currently drink & 96 & $67.13 \%$ & 92 & $74.19 \%$ & 0.2058 \\
\hline Currently smoke & 33 & $23.08 \%$ & 13 & $10.48 \%$ & 0.0053 \\
\hline Regularly exercise & 72 & $50.35 \%$ & 67 & $54.03 \%$ & 0.5489 \\
\hline Charlson comorbidity index (mean, SD) & 0.59 & 1.33 & 0.62 & 1.33 & 0.8712 \\
\hline \multicolumn{6}{|l|}{ Comorbidities } \\
\hline Depression & 22 & $15.38 \%$ & 13 & $10.48 \%$ & 0.232 \\
\hline Myocardial infarction & 13 & $9.09 \%$ & 10 & $8.06 \%$ & 0.7654 \\
\hline Stroke & 6 & $4.20 \%$ & 6 & $4.84 \%$ & 0.8018 \\
\hline TIA & 1 & $0.70 \%$ & 3 & $2.42 \%$ & 0.2677 \\
\hline Congestive heart failure & 4 & $2.80 \%$ & 4 & $3.23 \%$ & 0.8389 \\
\hline Hypertension & 64 & $44.76 \%$ & 46 & $37.10 \%$ & 0.2045 \\
\hline Angina & 18 & $12.59 \%$ & 9 & $7.26 \%$ & 0.1432 \\
\hline Arrhythmia & 4 & $2.80 \%$ & 9 & $7.26 \%$ & 0.1013 \\
\hline \multicolumn{6}{|l|}{ Prescribing physician } \\
\hline PCP & 27 & $18.88 \%$ & 26 & $20.97 \%$ & 0.6716 \\
\hline Endocrinologist/diabetologist & 113 & $79.02 \%$ & 94 & $75.81 \%$ & 0.5327 \\
\hline Nurse practitioner/physician assistant & 0 & $0.00 \%$ & 0 & $0.00 \%$ & N/A \\
\hline Other & 3 & $2.10 \%$ & 4 & $3.23 \%$ & 0.5718 \\
\hline Agree/strongly agree to being afraid of needles & 14 & $9.79 \%$ & 10 & $8.06 \%$ & 0.6217 \\
\hline \multicolumn{6}{|l|}{ Microvascular complications } \\
\hline Macular edema & 21 & $14.69 \%$ & 22 & $17.74 \%$ & 0.5013 \\
\hline Neuropathic pain & 31 & $21.68 \%$ & 22 & $17.74 \%$ & 0.4198 \\
\hline Kidney damage & 16 & $11.19 \%$ & 12 & $9.68 \%$ & 0.6871 \\
\hline End organ damage $(2010,2011$ only) & 7 & $7.29 \%$ & 7 & $8.05 \%$ & 0.8489 \\
\hline Foot ulcer & 9 & $6.29 \%$ & 5 & $4.03 \%$ & 0.4025 \\
\hline At least one microvascular complication & 58 & $40.56 \%$ & 46 & $37.10 \%$ & 0.5633 \\
\hline At least one complication (TIA, stroke, & 66 & $46.15 \%$ & 53 & $42.74 \%$ & 0.5766 \\
\hline \multicolumn{6}{|l|}{ HA, or microvascular complication) } \\
\hline \multicolumn{6}{|l|}{$\mathrm{HbA}_{\mathrm{Ic}}(\%)$} \\
\hline $\mathrm{HbA}_{\mathrm{Ic}}>7$ & 37 & $25.87 \%$ & 52 & $41.94 \%$ & 0.0057 \\
\hline $\mathrm{HbA}_{\mathrm{Ic}}$ (missing) & 59 & $41.26 \%$ & 35 & $28.23 \%$ & 0.025 \\
\hline \multicolumn{6}{|l|}{ Fasting glucose $(\mathrm{mg} / \mathrm{dl})$} \\
\hline Fasting glucose $>130$ & 25 & $17.48 \%$ & 17 & $13.71 \%$ & 0.3961 \\
\hline Fasting glucose (missing) & 83 & $58.04 \%$ & 67 & $54.03 \%$ & 0.5115 \\
\hline
\end{tabular}




\begin{tabular}{|c|c|c|c|c|c|}
\hline & \multicolumn{2}{|c|}{$\begin{array}{l}\text { Early insulin initiation } \\
(5 \text { years or less) } \\
n=143\end{array}$} & \multicolumn{2}{|c|}{$\begin{array}{l}\text { Late insulin initiation } \\
\text { (6 years or more) } \\
n=124\end{array}$} & \multirow[t]{2}{*}{$P$-value } \\
\hline & n & $\%$ & n & $\%$ & \\
\hline \multicolumn{6}{|l|}{ Satisfaction with treatment } \\
\hline Very/extremely satisfied with treatment & 80 & $56.74 \%$ & 66 & $53.23 \%$ & 0.5675 \\
\hline Very/extremely dissatisfied with treatment & 20 & $14.18 \%$ & 20 & $16.13 \%$ & 0.661 \\
\hline \multicolumn{6}{|l|}{ Morisky adherence } \\
\hline Compliant $($ MMAS = 0) & 82 & $85.42 \%$ & 69 & $79.31 \%$ & 0.2819 \\
\hline Forget to take medication & II & $11.46 \%$ & 14 & $16.09 \%$ & 0.3668 \\
\hline Careless about medication & 8 & $8.33 \%$ & 4 & $4.60 \%$ & 0.3029 \\
\hline Stop when feeling better & 0 & $0.00 \%$ & 1 & $1.15 \%$ & N/A \\
\hline Stop when feeling worse & 3 & $3.13 \%$ & 2 & $2.30 \%$ & 0.7312 \\
\hline
\end{tabular}

Abbreviations: BMI, body mass index; MMAS, Morisky Medication Adherence Scale; SD, standard deviation; HA, heart attack; TIA, transient ischemic attack; $\mathrm{PCP}$, primary care physician.

0.96, 95\% CI 0.93-0.991, $P=0.0108)$, and greater patient adherence (OR 1.82, 95\% CI 1.015-3.251, $P=0.0445$ ).

\section{Predictors of adherence to all types of treatment}

Using the MMAS (MMAS $=0$ ), the average age of type 2 diabetes patients adherent to therapy $(\mathrm{n}=385)$ was higher than that of those nonadherent to therapy $(\mathrm{n}=121,60.72$ years versus 57.01 years, respectively, $P=0.0008$, Table 9). The adherent patients were older at diagnosis ( 47.85 years versus 45.28 years, $P=0.0209$ ), while the adherent group was also more likely to be female than the nonadherent group (36.88\% versus $26.45 \%, P=0.0282$ ). Socioeconomically, the adherent group was more likely to have an income of $€ 20,000$ to $<€ 50,000$ ( $55.84 \%$ versus $43.80 \%, P=0.0214$ ), as well as being less likely to be employed ( $23.38 \%$ versus $36.36 \%, P=0.0087$ ), and were more frequently using insulin (39.48\% versus $26.45 \%, P=0.0066$ ). In terms of clinical characteristics, the adherent group was less likely to drink alcohol $(P=0.0365)$, had significantly more myocardial infarction $(P=0.0473)$, and had more macular edema complications $(P=0.0005)$. They were more frequently followed up by an endocrinologist or diabetologist $(47.53 \%$ versus $34.71 \%, P=0.0118)$. We did not see any difference in terms of control of diabetes.

A logistic regression showed two significant factors of adherence, ie, older age (OR 1.04, 95\% CI 1.011-1.071, $P=0.0075$, Table 10) and macular edema or diabetic retinopathy (OR 4.282, 95\% CI 1.171-15.659, $P=0.0279$ ). Conversely, currently drinking alcohol (OR $0.559,95 \% \mathrm{CI}$ $0.319-0.982, P=0.0429$ ), and $\mathrm{HbA}_{1 \mathrm{c}}>7 \%$ (OR $0.551,95 \%$ CI $0.307-0.989, P=0.0458$ ) were significant predictors of nonadherence.

Insulin did not appear to be a determining factor of adherence when insulin users were compared with all users of oral bitherapy or tritherapy. However, as shown in Table 10, logistic regression comparing insulin users with uncontrolled oral bitherapy and tritherapy users $(n=256)$ showed that insulin

Table 6 Statistically significant factors influencing early insulin initiation

\begin{tabular}{|c|c|c|c|c|c|c|c|}
\hline Parameter & Estimate & OR & 95\% LCL for OR & 95\% UCL for OR & SE & Chi-square & $P$-value \\
\hline \multicolumn{8}{|c|}{ Influencing early insulin initiation versus late initiation (median split $=5, n=265$ ) } \\
\hline Age at diagnosis & 0.0309 & 1.031 & 1.005 & 1.059 & 0.0132 & 5.4509 & 0.0196 \\
\hline Income: & -0.795 & 0.452 & 0.239 & 0.856 & 0.3259 & 5.944 & 0.0148 \\
\hline \multicolumn{8}{|l|}{$€ 20,000$ to $<€ 50,000$} \\
\hline Currently smoking & 0.9308 & 2.537 & 1.165 & 5.524 & 0.3971 & 5.496 & 0.0191 \\
\hline $\mathrm{HbA}_{\mathrm{Ic}}>7 \%$ & -0.6 & 0.549 & 0.28 & 1.076 & 0.3436 & 3.0503 & 0.0807 \\
\hline \multicolumn{8}{|c|}{ Influencing early insulin initiation versus uncontrolled bitherapy or tritherapy $(n=245)$} \\
\hline $\begin{array}{l}\text { Income: } \\
€ 20,000 \text { to }<€ 50,000\end{array}$ & -0.825 & 0.438 & 0.195 & 0.985 & 0.4133 & 3.9838 & 0.0459 \\
\hline $\begin{array}{l}\text { Income: } \\
\geq € 50,000\end{array}$ & -1.347 & 0.26 & 0.072 & 0.94 & 0.6556 & 4.2236 & 0.0399 \\
\hline Prescribing physician: GP & -2.292 & 0.101 & 0.05 & 0.204 & 0.3577 & 41.059 & $<0.0001$ \\
\hline
\end{tabular}

Abbreviations: OR, odds ratio; LCL, lower confidence limit; UCL, upper confidence limit; SE, standard error; GP, general practitioner. 
Table 7 Analyzed population: all controlled insulin/bitherapy or tritherapy users versus uncontrolled insulin/bitherapy or tritherapy users (excluding missing $\mathrm{HbA}_{\mathrm{Ic}}$ level)

\begin{tabular}{|c|c|c|c|c|c|}
\hline & \multicolumn{2}{|c|}{$\begin{array}{l}\text { All controlled insulin/ } \\
\text { bi- or tritherapy users } \\
n=224\end{array}$} & \multicolumn{2}{|c|}{$\begin{array}{l}\text { Uncontrolled insulin/ } \\
\text { bi- or tritherapy users (excluding } \\
\text { missing } \mathrm{HbA}_{\mathrm{Ic}} \text { level) } \\
\mathrm{n}=\mathbf{2 0 8}\end{array}$} & \multirow[t]{2}{*}{$P$-value } \\
\hline & $\mathbf{n}$ & $\%$ & $\mathbf{n}$ & $\%$ & \\
\hline Age (mean, SD) & 60.93 & 10.5 & 59.54 & 9.16 & 0.1413 \\
\hline Age at diagnosis & 48.16 & $1 \mathrm{I} .57$ & 45.38 & 10.92 & 0.0109 \\
\hline Age at insulin initiation (only insulin users) & 50.72 & 15.23 & 51.17 & 12.03 & 0.8273 \\
\hline Years diagnosed & 12.95 & 9.48 & 14.09 & 8.81 & 0.2009 \\
\hline \multicolumn{6}{|l|}{ Therapy } \\
\hline $\mathrm{Bi} /$ tri oral therapy users & 137 & $61.16 \%$ & 105 & $50.48 \%$ & 0.0254 \\
\hline Insulin users & 87 & $38.84 \%$ & 103 & $49.52 \%$ & 0.0254 \\
\hline \multicolumn{6}{|l|}{ Sex } \\
\hline Male & 147 & $65.63 \%$ & 141 & $67.79 \%$ & 0.634 \\
\hline Female & 77 & $34.38 \%$ & 67 & $32.21 \%$ & 0.634 \\
\hline Currently employed & 51 & $22.77 \%$ & 64 & $30.77 \%$ & 0.0609 \\
\hline \multicolumn{6}{|l|}{ Household income } \\
\hline$<20,000 €$ & 56 & $25.00 \%$ & 50 & $24.04 \%$ & 0.8167 \\
\hline $20,000 €$ to $<50,000 €$ & 119 & $53.13 \%$ & 116 & $55.77 \%$ & 0.5819 \\
\hline $50,000 €$ or more & 36 & $16.07 \%$ & 25 & $12.02 \%$ & 0.2252 \\
\hline Decline to answer & 13 & $5.80 \%$ & 17 & $8.17 \%$ & 0.3364 \\
\hline College education & 77 & $34.38 \%$ & 66 & $31.73 \%$ & 0.5598 \\
\hline \multicolumn{6}{|l|}{ BMI } \\
\hline Underweight & I & $0.45 \%$ & 0 & $0.00 \%$ & $\mathrm{~N} / \mathrm{A}$ \\
\hline Normal weight & 23 & $10.27 \%$ & 28 & $13.46 \%$ & 0.3066 \\
\hline Overweight & 90 & $40.18 \%$ & 80 & $38.46 \%$ & 0.7155 \\
\hline Obese & 105 & $46.88 \%$ & 96 & $46.15 \%$ & 0.8808 \\
\hline Decline to answer & 5 & $2.23 \%$ & 4 & $1.92 \%$ & 0.822 \\
\hline \multicolumn{6}{|l|}{ Health behaviors } \\
\hline Currently drink & 175 & $78.13 \%$ & 152 & $73.08 \%$ & 0.2232 \\
\hline Currently smoke & 29 & $12.95 \%$ & 36 & $17.31 \%$ & 0.2076 \\
\hline Regularly exercise & 137 & $61.16 \%$ & 109 & $52.40 \%$ & 0.0665 \\
\hline Charlson comorbidity index (mean, SD) & 0.38 & 0.91 & 0.44 & 0.87 & 0.4965 \\
\hline \multicolumn{6}{|l|}{ Comorbidities } \\
\hline Depression & 25 & $11.16 \%$ & 25 & $12.02 \%$ & 0.7811 \\
\hline Myocardial infarction & 15 & $6.70 \%$ & 10 & $4.81 \%$ & 0.3988 \\
\hline Stroke & 6 & $2.68 \%$ & 10 & $4.81 \%$ & 0.2469 \\
\hline TIA & 2 & $0.89 \%$ & 4 & $1.92 \%$ & 0.3676 \\
\hline Congestive heart failure & 3 & $1.34 \%$ & 7 & $3.37 \%$ & 0.1686 \\
\hline Hypertension & 106 & $47.32 \%$ & 95 & $45.67 \%$ & 0.7319 \\
\hline Angina & 28 & $12.50 \%$ & 17 & $8.17 \%$ & 0.1388 \\
\hline Arrhythmia & 11 & $4.91 \%$ & 18 & $8.65 \%$ & 0.124 \\
\hline \multicolumn{6}{|l|}{ Prescribing physician } \\
\hline PCP & 104 & $46.43 \%$ & 95 & $45.67 \%$ & 0.8751 \\
\hline Endocrinologist/diabetologist & 117 & $52.23 \%$ & 112 & $53.85 \%$ & 0.7374 \\
\hline Nurse practitioner/physician assistant & 0 & $0.00 \%$ & 0 & $0.00 \%$ & $\mathrm{~N} / \mathrm{A}$ \\
\hline Other & 3 & $1.34 \%$ & I & $0.48 \%$ & 0.3442 \\
\hline Agree/strongly agree to being afraid of needles & 21 & $9.38 \%$ & 22 & $10.58 \%$ & 0.6778 \\
\hline \multicolumn{6}{|l|}{ Microvascular complications } \\
\hline Macular edema & 14 & $6.25 \%$ & 30 & $14.42 \%$ & 0.0055 \\
\hline Neuropathic pain & 26 & $11.61 \%$ & 33 & $15.87 \%$ & 0.2003 \\
\hline Kidney damage & 10 & $4.46 \%$ & 16 & $7.69 \%$ & 0.1628 \\
\hline End organ damage $(2010,2011$ only) & 7 & $3.70 \%$ & 5 & $3.38 \%$ & 0.8725 \\
\hline Foot ulcer & 6 & $2.68 \%$ & 11 & $5.29 \%$ & 0.1685 \\
\hline At least one microvascular complication & 45 & $20.09 \%$ & 70 & $33.65 \%$ & 0.0015 \\
\hline
\end{tabular}




\begin{tabular}{|c|c|c|c|c|c|}
\hline & \multicolumn{2}{|c|}{$\begin{array}{l}\text { All controlled insulin/ } \\
\text { bi- or tritherapy users } \\
\mathbf{n}=\mathbf{2 2 4}\end{array}$} & \multicolumn{2}{|c|}{$\begin{array}{l}\text { Uncontrolled insulin/ } \\
\text { bi- or tritherapy users (excluding } \\
\text { missing } \mathrm{Hba}_{1 \mathrm{l}} \text { level) } \\
\mathbf{n}=\mathbf{2 0 8}\end{array}$} & \multirow[t]{2}{*}{$P$-value } \\
\hline & $\mathbf{n}$ & $\%$ & $\mathbf{n}$ & $\%$ & \\
\hline $\begin{array}{l}\text { At least one complication (TIA, stroke, HA, or } \\
\text { microvascular complication) }\end{array}$ & 54 & $24.11 \%$ & 81 & $38.94 \%$ & 0.0009 \\
\hline \multicolumn{6}{|l|}{$\mathrm{HbA}_{\mathrm{Ic}}(\%)$} \\
\hline $\mathrm{HbA}_{\mathrm{lc}}>7$ & 0 & $0.00 \%$ & 167 & $80.29 \%$ & N/A \\
\hline $\mathrm{HbA}_{\mathrm{Ic}}$ (missing) & 0 & $0.00 \%$ & 41 & $19.71 \%$ & N/A \\
\hline \multicolumn{6}{|l|}{ Fasting glucose (mg/dl) } \\
\hline Fasting glucose $>130$ & 19 & $8.48 \%$ & 68 & $32.69 \%$ & $<0.0001$ \\
\hline Fasting glucose (missing) & 130 & $58.04 \%$ & 86 & $41.35 \%$ & 0.0005 \\
\hline \multicolumn{6}{|l|}{ Satisfaction with treatment } \\
\hline Very/extremely satisfied with treatment & 146 & $65.18 \%$ & 97 & $46.63 \%$ & $<0.0001$ \\
\hline Very/extremely dissatisfied with treatment & 31 & $13.84 \%$ & 24 & $11.54 \%$ & 0.4728 \\
\hline \multicolumn{6}{|l|}{ Morisky adherence $(2010,2011$ only $)$} \\
\hline Compliant $(\mathrm{MMAS}=0)$ & 154 & $81.48 \%$ & 108 & $72.97 \%$ & 0.0667 \\
\hline Forget to take medication & 30 & $15.87 \%$ & 37 & $25.00 \%$ & 0.0411 \\
\hline Careless about medication & 14 & $7.41 \%$ & 12 & $8.11 \%$ & 0.8123 \\
\hline Stop when feeling better & 2 & $1.06 \%$ & 0 & $0.00 \%$ & N/A \\
\hline Stop when feeling worse & 4 & $2.12 \%$ & 5 & $3.38 \%$ & 0.4885 \\
\hline
\end{tabular}

Abbreviations: BMI, body mass index; MMAS, Morisky Medication Adherence Scale; SD, standard deviation; HA, heart attack; TIA, transient ischemic attack; $\mathrm{PCP}$, primary care physician.

users had 3.0 times greater odds of being adherent (OR $2.983,95 \%$ CI $1.37-6.495, P=0.0059)$, with even greater odds when early insulin users were considered $(3.337,95 \%$ CI $1.295-8.595, P=0.0126)$.

\section{Impact of insulin on quality of life}

Linear regression models were used to assess the impact of insulin, early insulin initiation, and short ( $\leq 5$ years) insulin duration versus uncontrolled bitherapy and tritherapy users on MCS and PCS scores. Table 11 summarizes the adjusted means for MCS and PCS. Overall, no significant difference was observed between the two groups on the MCS (44.182 versus 45.832), and PCS (39.611 versus 40.093) scores. However, one must note the significant difference $(P=0.0304)$ on the MCS in the early insulin initiation subgroup, which can be explained by the eventual complications and negative perception of insulin as a last resort treatment. Indeed, there were no significant differences between the early insulin patients with no complications versus patients uncontrolled by bitherapy or tritherapy. As shown in Figure 1, when the presence or absence of complications were considered, whatever the treatment, the presence of complications had a negative impact on both MCS (42.66 versus 47.36, $P<0.0001)$ and PCS (34.32 versus 43.48, $P<0.0001)$.

Analysis of MCS and PCS data based on duration of insulin therapy ( $<3$ years, $3-5$ years, $6-10$ years, $\geq 11$ years) allowed observation of the stability of quality of life scores on the two dimensions, with the only significant difference on physical health decreasing after 11 years and more of treatment, compared with $<3$ years in relation to the appearance of chronic complications (Figure 2).

Table 8 Statistically significant factors influencing control of diabetes: all controlled insulin/bitherapy or tritherapy users versus uncontrolled insulin/bitherapy or tritherapy users $(n=336)$

\begin{tabular}{llllllll}
\hline Parameter & Estimate & OR & 95\% LCL for OR & 95\% UCL for OR & SE & Chi-square & $\boldsymbol{P}$-value \\
\hline Duration of type 2 diabetes & -0.041 & 0.96 & 0.93 & 0.991 & 0.0161 & 6.4939 & 0.0108 \\
Compliant & 0.5967 & 1.816 & 1.015 & 3.251 & 0.297 & 4.0362 & 0.0445 \\
$\begin{array}{l}\text { Very/extremely satisfied } \\
\text { with treatment }\end{array}$ & 0.934 & 2.545 & 1.556 & 4.16 & 0.2508 & 13.8687 & 0.0002 \\
\hline
\end{tabular}

Note: Excludes respondents who did not know their $\mathrm{HbA}_{\mathrm{lc}}$ level.

Abbreviations: OR, odds ratio; LCL, lower confidence limit; UCL, upper confidence limit; SE, standard error. 
Table 9 Analyzed population: all adherent insulin/bitherapy or tritherapy users versus all nonadherent insulin/bitherapy or tritherapy users

\begin{tabular}{|c|c|c|c|c|c|}
\hline & \multicolumn{2}{|c|}{$\begin{array}{l}\text { Non-compliant - total } \\
\text { treatment insulin/ } \\
\text { bi- or tritherapy users } \\
n=I 2 I \\
\end{array}$} & \multicolumn{2}{|c|}{$\begin{array}{l}\text { Compliant - total } \\
\text { treatment insulin/ } \\
\text { bi- or tritherapy users } \\
\mathbf{n}=\mathbf{3 8 5} \\
\end{array}$} & \multirow[t]{2}{*}{$P$-value } \\
\hline & $\mathbf{n}$ & $\%$ & $\mathbf{n}$ & $\%$ & \\
\hline Age (mean, SD) & 57.01 & 10.75 & 60.72 & 9.27 & 0.0008 \\
\hline Age at diagnosis & 45.28 & 10.58 & 47.85 & 10.68 & 0.0209 \\
\hline Age at insulin initiation (only insulin users) & 50.47 & 11.82 & 52.51 & 12.02 & 0.3813 \\
\hline Years diagnosed & 11.73 & 8.2 & 12.97 & 8.82 & 0.1564 \\
\hline \multicolumn{6}{|l|}{ Therapy } \\
\hline $\mathrm{Bi} /$ tri oral therapy users & 89 & $73.55 \%$ & 233 & $60.52 \%$ & 0.0066 \\
\hline Insulin users & 32 & $26.45 \%$ & 152 & $39.48 \%$ & 0.0066 \\
\hline \multicolumn{6}{|l|}{ Sex } \\
\hline Male & 89 & $73.55 \%$ & 243 & $63.12 \%$ & 0.0282 \\
\hline Female & 32 & $26.45 \%$ & 142 & $36.88 \%$ & 0.0282 \\
\hline Currently employed & 44 & $36.36 \%$ & 90 & $23.38 \%$ & 0.0087 \\
\hline \multicolumn{6}{|l|}{ Household income } \\
\hline$<20,000 €$ & 34 & $28.10 \%$ & 99 & $25.71 \%$ & 0.6091 \\
\hline $20,000 €$ to $<50,000 €$ & 53 & $43.80 \%$ & 215 & $55.84 \%$ & 0.0214 \\
\hline $50,000 €$ or more & 19 & $15.70 \%$ & 36 & $9.35 \%$ & 0.082 \\
\hline Decline to answer & 15 & $12.40 \%$ & 35 & $9.09 \%$ & 0.3232 \\
\hline College education & 47 & $38.84 \%$ & $14 \mid$ & $36.62 \%$ & 0.6619 \\
\hline \multicolumn{6}{|l|}{ BMI } \\
\hline Underweight & 0 & $0.00 \%$ & I & $0.26 \%$ & N/A \\
\hline Normal weight & 14 & $11.57 \%$ & 38 & $9.87 \%$ & 0.6051 \\
\hline Overweight & 41 & $33.88 \%$ & 145 & $37.66 \%$ & 0.4475 \\
\hline Obese & 64 & $52.89 \%$ & 186 & $48.31 \%$ & 0.3801 \\
\hline Decline to answer & 2 & $1.65 \%$ & 15 & $3.90 \%$ & 0.1428 \\
\hline \multicolumn{6}{|l|}{ Health behaviors } \\
\hline Currently drink & 96 & $79.34 \%$ & 270 & $70.13 \%$ & 0.0365 \\
\hline Currently smoke & 24 & $19.83 \%$ & 60 & $15.58 \%$ & 0.298 \\
\hline Regularly exercise & 59 & $48.76 \%$ & 202 & $52.47 \%$ & 0.4777 \\
\hline Charlson comorbidity index (mean, SD) & 0.29 & 0.72 & 0.36 & 0.72 & 0.3776 \\
\hline \multicolumn{6}{|l|}{ Comorbidities } \\
\hline Depression & 10 & $8.26 \%$ & 50 & $12.99 \%$ & 0.1218 \\
\hline Myocardial infarction & 3 & $2.48 \%$ & 24 & $6.23 \%$ & 0.0473 \\
\hline Stroke & 3 & $2.48 \%$ & 15 & $3.90 \%$ & 0.4124 \\
\hline TIA & 2 & $1.65 \%$ & 3 & $0.78 \%$ & 0.4831 \\
\hline Congestive heart failure & 0 & $0.00 \%$ & 10 & $2.60 \%$ & N/A \\
\hline Hypertension & 58 & $47.93 \%$ & 164 & $42.60 \%$ & 0.3059 \\
\hline Angina & 21 & $17.36 \%$ & 44 & $11.43 \%$ & 0.1217 \\
\hline Arrhythmia & 4 & $3.31 \%$ & 28 & $7.27 \%$ & 0.0606 \\
\hline \multicolumn{6}{|l|}{ Prescribing physician } \\
\hline $\mathrm{PCP}$ & 79 & $65.29 \%$ & 199 & $51.69 \%$ & 0.0077 \\
\hline Endocrinologist/diabetologist & 42 & $34.71 \%$ & 183 & $47.53 \%$ & 0.0118 \\
\hline Nurse practitioner/physician assistant & 0 & $0.00 \%$ & 0 & $0.00 \%$ & N/A \\
\hline Other & 0 & $0.00 \%$ & 3 & $0.78 \%$ & $N / A$ \\
\hline Agree/strongly agree to being afraid of needles & 16 & $13.22 \%$ & 34 & $8.83 \%$ & 0.1989 \\
\hline \multicolumn{6}{|l|}{ Microvascular complications } \\
\hline Macular edema & 3 & $2.48 \%$ & 38 & $9.87 \%$ & 0.0005 \\
\hline Neuropathic pain & 16 & $13.22 \%$ & 45 & $11.69 \%$ & 0.6605 \\
\hline Kidney damage & 4 & $3.31 \%$ & 19 & $4.94 \%$ & 0.4083 \\
\hline End organ damage $(2010,201$ I only $)$ & 4 & $3.31 \%$ & 15 & $3.90 \%$ & 0.7566 \\
\hline Foot ulcer & 4 & $3.31 \%$ & 15 & $3.90 \%$ & 0.7566 \\
\hline At least one microvascular complication & 25 & $20.66 \%$ & 93 & $24.16 \%$ & 0.4154 \\
\hline $\begin{array}{l}\text { At least one complication (TIA, stroke, HA, } \\
\text { or microvascular complication) }\end{array}$ & 30 & $24.79 \%$ & 110 & $28.57 \%$ & 0.4078 \\
\hline
\end{tabular}


Table 9 (Continued)

\begin{tabular}{|c|c|c|c|c|c|}
\hline & \multicolumn{2}{|c|}{$\begin{array}{l}\text { Non-compliant - total } \\
\text { treatment insulin/ } \\
\text { bi- or tritherapy users } \\
n=|2|\end{array}$} & \multicolumn{2}{|c|}{$\begin{array}{l}\text { Compliant - total } \\
\text { treatment insulin/ } \\
\text { bi- or tritherapy users } \\
n=385\end{array}$} & \multirow[t]{2}{*}{$P$-value } \\
\hline & $\mathbf{n}$ & $\%$ & $\mathbf{n}$ & $\%$ & \\
\hline \multicolumn{6}{|l|}{$\mathrm{HbA}_{\mathrm{Ic}}(\%)$} \\
\hline $\mathrm{HbA}_{\mathrm{Ic}}>7$ & 39 & $32.23 \%$ & 104 & $27.01 \%$ & 0.2802 \\
\hline $\mathrm{HbA}_{\mathrm{Ic}}$ (missing) & 47 & $38.84 \%$ & 127 & $32.99 \%$ & 0.247 \\
\hline \multicolumn{6}{|l|}{ Fasting glucose (mg/dl) } \\
\hline Fasting glucose $>130$ & 5 & $4.13 \%$ & 15 & $3.90 \%$ & 0.9089 \\
\hline Fasting glucose (missing) & 80 & $66.12 \%$ & 243 & $63.12 \%$ & 0.546 \\
\hline \multicolumn{6}{|l|}{ Satisfaction with treatment } \\
\hline Very/extremely satisfied with treatment & 56 & $46.67 \%$ & 218 & $56.77 \%$ & 0.0545 \\
\hline Very/extremely dissatisfied with treatment & 13 & $10.83 \%$ & 55 & $14.32 \%$ & 0.2998 \\
\hline
\end{tabular}

Abbreviations: BMI, body mass index; SD, standard deviation; HA, heart attack; TIA, transient ischemic attack; PCP, primary care physician.

Table I 0 Statistically significant factors influencing adherence to diabetes medication: adherent versus nonadherent users

\begin{tabular}{|c|c|c|c|c|c|c|c|}
\hline Parameter & Estimate & OR & 95\% LCL for OR & 95\% UCL for OR & SE & Chi-square & $P$-value \\
\hline \multicolumn{8}{|c|}{ All insulin/bitherapy or tritherapy users $(n=503)$} \\
\hline Age at diagnosis & 0.0396 & 1.04 & 1.011 & I.07। & 0.0148 & 7.1376 & 0.0075 \\
\hline Currently drinking alcohol & -0.5813 & 0.559 & 0.319 & 0.982 & 0.2871 & 4.0984 & 0.0429 \\
\hline $\begin{array}{l}\text { Macular edema or diabetic } \\
\text { retinopathy }\end{array}$ & 1.4543 & 4.282 & 1.171 & 15.659 & 0.6616 & 4.8316 & 0.0279 \\
\hline $\mathrm{HbA}_{\mathrm{Ic}}>7 \%$ & -0.5954 & 0.551 & 0.307 & 0.989 & 0.2981 & 3.9884 & 0.0458 \\
\hline \multicolumn{8}{|c|}{ All insulin users and uncontrolled bitherapy or tritherapy users $(n=256)$} \\
\hline $\begin{array}{l}\text { All insulin versus uncontrolled } \\
\text { bitherapy or tritherapy }\end{array}$ & 1.0931 & 2.983 & 1.37 & 6.495 & 0.3969 & 7.5828 & 0.0059 \\
\hline $\begin{array}{l}\text { Macular edema or diabetic } \\
\text { retinopathy }\end{array}$ & 1.8705 & 6.492 & 1.301 & 32.381 & 0.8199 & 5.2042 & 0.0225 \\
\hline $\begin{array}{l}\text { Very/extremely satisfied } \\
\text { with treatment }\end{array}$ & $0.7|4|$ & 2.042 & 1.043 & 3.998 & 0.3427 & 4.3427 & 0.0372 \\
\hline \multicolumn{8}{|c|}{ Early insulin initiation and uncontrolled bitherapy or tritherapy users $(n=169)$} \\
\hline $\begin{array}{l}\text { Early insulin versus uncontrolled } \\
\text { bitherapy or tritherapy }\end{array}$ & 1.205 & 3.337 & 1.295 & 8.595 & 0.4828 & 6.2298 & 0.0126 \\
\hline
\end{tabular}

Abbreviations: OR, odds ratio; LCL, lower confidence limit; UCL, upper confidence limit; SE, standard error.

Table I I Summary of adjusted means for MCS and PCS scores on bitherapy or tritherapy

\begin{tabular}{|c|c|c|c|}
\hline & All insulin users & $\begin{array}{l}\text { Uncontrolled bitherapy } \\
\text { or tritherapy users }\end{array}$ & $P$-value \\
\hline \multicolumn{4}{|l|}{ Adjusted means } \\
\hline Mental component summary & 44.18 & 45.83 & 0.2317 \\
\hline \multirow[t]{2}{*}{ Physical component summary } & 39.61 & 40.09 & 0.6686 \\
\hline & Early insulin initiation ( $\leq 5$ years) & $\begin{array}{l}\text { Uncontrolled bitherapy or } \\
\text { tritherapy users }\end{array}$ & \\
\hline Mental component summary & 42.85 & 46.37 & 0.0304 \\
\hline \multirow[t]{2}{*}{ Physical component summary } & 39.49 & 40.35 & 0.5232 \\
\hline & $\begin{array}{l}\text { Early insulin initiation ( } \leq 5 \text { years) } \\
\text { with no complications }\end{array}$ & $\begin{array}{l}\text { Uncontrolled bitherapy or } \\
\text { tritherapy users }\end{array}$ & \\
\hline Mental component summary & 44.88 & 46.71 & 0.2945 \\
\hline Physical component summary & 42.94 & 42.03 & 0.5748 \\
\hline
\end{tabular}

Note: Data in bold is significant.

Abbreviations: MCS, Mental Component Summary; PCS, Physical Component Summary. 


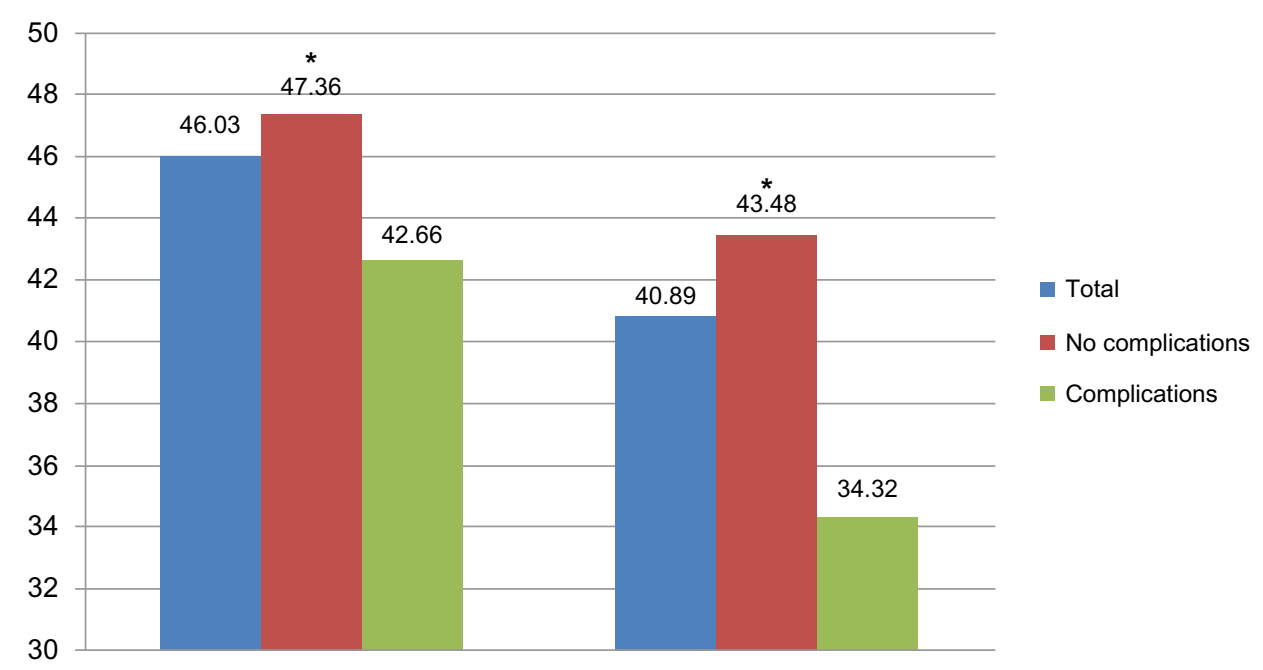

Figure I Mean MCS and PCS scores by type 2 diabetes complications (insulin users).

Note: $* P<0.0001$ for no complications versus complications.

Abbreviations: MCS, Mental Component Summary; PCS, Physical Component Summary.

Also, as post hoc analyses, type 2 diabetes quality of life scores were compared with other disease conditions. Figure 3 shows that patients with type 2 diabetes have lower mental health scores $(\mathrm{MCS}=45.86)$ relative to the average person, but higher levels of mental health than people diagnosed with depression, and similar scores relative to patients with metabolic syndrome, allergic rhinitis, or hepatitis C. Patients with type 2 diabetes had lower levels of physical health (PCS $=42.32$ ) relative to the average person and people suffering from allergic rhinitis and depression, but very similar levels of physical health relative to patients with hepatitis C. Also, quite surprisingly, they had higher levels of physical health compared with those having metabolic syndrome.

\section{Discussion}

\section{Determinants of insulin prescription}

In this study, factors determining insulin prescription in multivariable analysis consisted of lower age at type 2 diabetes diagnosis, the presence of retinopathy, neuropathic pain, being treated by a specialist, and lower $(<€ 50,000$ per annum) income. Factors determining early insulin prescription in the course of the disease, as compared with late insulin prescription, were of younger age at diagnosis

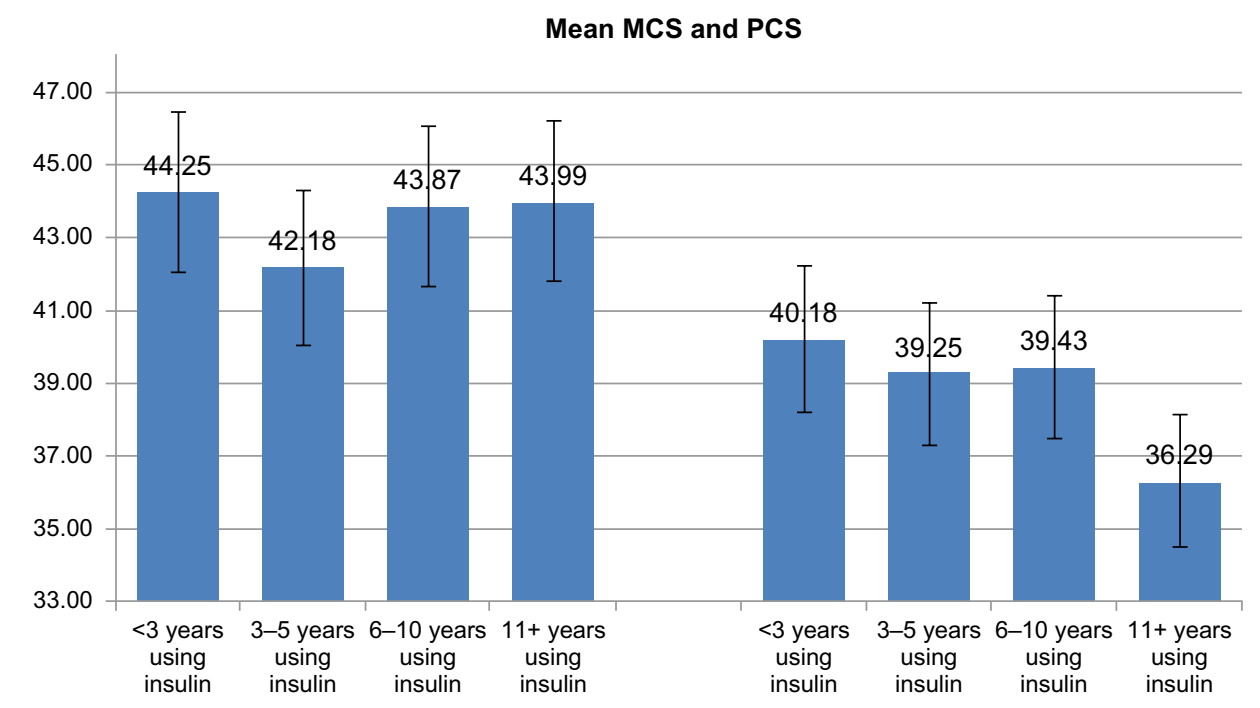

Figure 2 Mean MCS (left) and PCS scores (right): comparison of early versus late insulin initiation.

Abbreviations: MCS, Mental Component Summary; PCS, Physical Component Summary. 


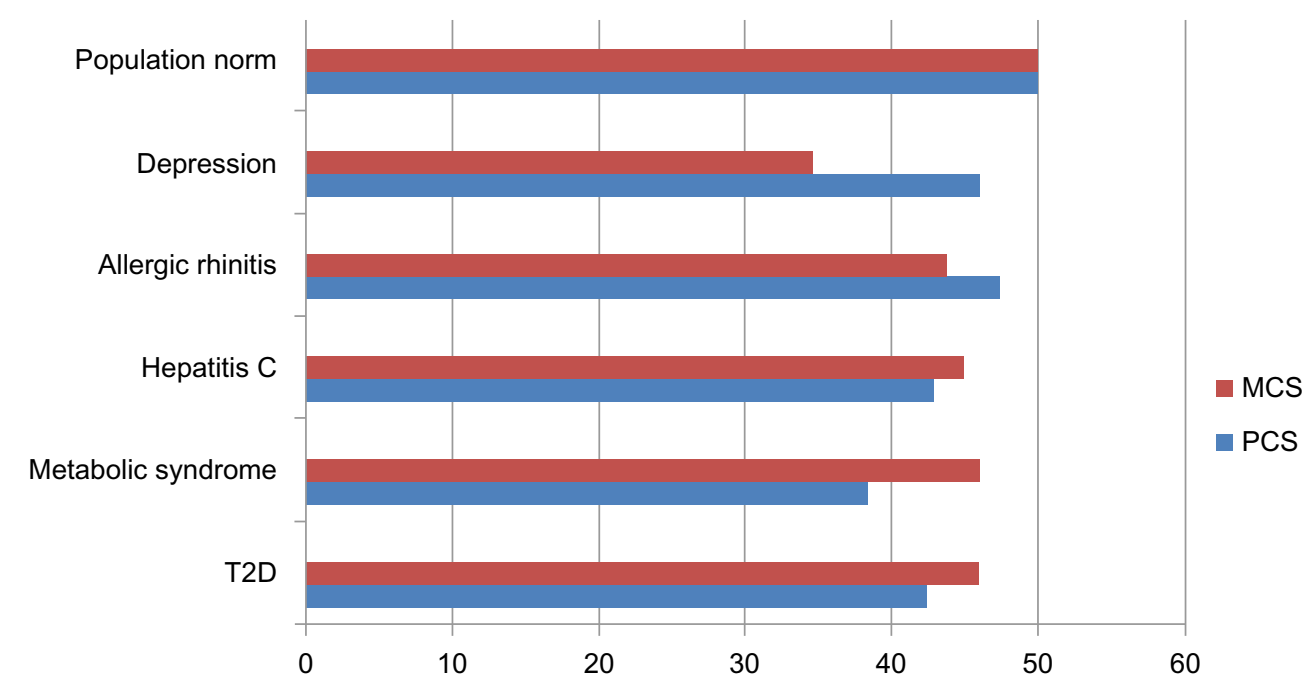

Figure 3 PCS and MCS scores for common conditions in Europe.

Abbreviations: MCS, Mental Component Summary; PCS, Physical Component Summary; T2D, type 2 diabetes.

and had a lower income. When the factors determining early insulin prescription in the course of the disease were analyzed as compared with uncontrolled oral therapy, lower income and being treated by a specialist were observed to be significant.

It is not surprising to observe that patients with severe complications are more often treated with insulin; the effect of an early diabetes onset may be explained by the presence of late autoimmune diabetes among the patients in the study, with up to $10 \%$ of patients diagnosed with type 2 diabetes found to have anti-glutamic acid decarboxylase antibodies. ${ }^{26}$ The higher frequency of specialist care in insulin treated patients must be interpreted with caution. It does not mean that general practitioners are reluctant to prescribe insulin. They may refer the patient to the specialist when they appropriately estimate that insulin should be prescribed: incidentally, this may explain why, in one study, clinical inertia concerning insulin prescription was found to be more frequent among general practitioners than among specialists. ${ }^{14}$

The independent effect of patients' income observed herein is more original: our data suggest that patients with a lower income are more frequently treated by insulin. While the deleterious effect of social deprivation on patient adherence is known, ${ }^{27}$ whether low income leads to an increased risk of doctors' clinical inertia is harder to determine. For instance, one study showed that patients of low socioeconomic class had diabetes more often and were able to achieve treatment targets less often, but in fact had indicators of good practice more often, ie, measurement of $\mathrm{HbA}_{1 \mathrm{c}}$, microalbuminuria, eye examination, treatment by insulin in insufficient control of diabetes. ${ }^{28}$ However, a more recent study did not show evidence of a language barrier effect on intensification of therapy in patients with type 2 diabetes imbalance, but a low level of income was clearly associated with less treatment intensification. ${ }^{29}$

\section{Predictors of control of diabetes}

In the bivariate analysis, we observed classical determinants of diabetes control, such as diabetes duration, adherence to therapy (the effect of adherence on metabolic control, hypercholesterolemia, and hypertension is also well substantiated), ${ }^{30-33}$ and, as expected, we observed an association of good control with less frequency of diabetic complications.

The strong effect of satisfaction towards treatment is more puzzling: not the fact that controlled patients are more frequently satisfied by their treatment, which seems to hold true. But the fact that in the multivariable analysis this determinant had by far the strongest link with diabetes control, suggesting that other factors (eg, dosage, number of required treatments per day) could be influencing this particular variable.

\section{Predictors of patient adherence}

This study confirms the known determinants of adherence observed in the multivariable analysis, ie, older age, ${ }^{34-37}$ abstinence from drinking, ${ }^{38}$ metabolic control, ${ }^{30,31,39}$ and the presence of complications ${ }^{37}$ Surprisingly, we did not observe any association between nonsmoking and adherence, which was shown in some studies. ${ }^{40-43}$ In bivariate analysis, we observed that adherent respondents were less likely to be employed. This was also observed in the recent French ENTRED (Medication Adherence in Type 2 Diabetes) study. ${ }^{37}$ In our 
study, adherent patients reported lower income status, while in the ENTRED study, ${ }^{37}$ financial difficulties were associated with a low adherence rate, as in a Swedish study. ${ }^{27}$ The fact that, in our study, lower income was associated with good adherence is consistent with a Canadian study. ${ }^{44}$ The fact that the same findings were observed in France, where diabetic drugs are paid by the social security program, suggests that this effect may not be due to what was observed in Canada (the effect of copayment).

In our study, insulin adherence was better in patients treated with insulin than in those treated with oral antidiabetic medication, especially in the case of early insulin prescription. Indeed, insulin users had 3.0 times greater odds of being adherent compared with uncontrolled oral bitherapy or tritherapy users (OR 2.983, $P=0.0059$ ). Interestingly, the fact that adherence may be better with injections was proposed as an argument to favor injectable rather than oral penicillin in children with impaired splenic function, ${ }^{45}$ and it is also a concern when considering adherence to cancer therapy. ${ }^{46}$ This better adherence to injectable therapy, observed in our study, is in contradiction with the general concern of physicians concerning patient adherence as a cause of psychological insulin resistance. ${ }^{47}$

\section{Effect of treatment with insulin on quality of life}

Overall, no significant difference was observed between insulin users and uncontrolled bitherapy or tritherapy users concerning MCS and PCS scores. Quality of life, both physical and mental, was therefore not altered compared with that in patients uncontrolled on bitherapy or tritherapy. Physical health scores decreased after 11 years of diabetes therapy, possibly an effect of the appearance of chronic complications. The lower MCS score in the early insulin initiation subgroup may also be explained by the eventual presence of complications, which were well analyzed in this study (Figure 1). Indeed, there were no significant differences for the early insulin patients with no complications versus patients uncontrolled by bitherapy or tritherapy.

In this context of quality of life, reflecting the burden of the disease, it was interesting to compare the European data concerning type 2 diabetes with those of other highprevalence chronic diseases. For this comparison, previous European NHWS studies were prioritized, because the methodology and measures were the same, thus providing the most suitable and relevant basis for comparison with diabetes in the current study. Comparison of scores (Figure 3) shows that the MCS and PCS scores for patients with type 2 diabetes are comparable across other conditions, and patients with type 2 diabetes have relative lower scores than the general population.

Although this study has some weak points, many of the findings are consistent with those reported in the literature. The first limitation is the relatively small number of patients as compared with other studies addressing specific issues, such as patient adherence based on refill evaluation, allowing analysis of much larger populations. Thus, the small sample sizes in the current study precluded the ability to conduct multivariable analyses for specific delays or duration of treatment, or to generalize broadly from the current data. Future research should adjust for possible confounds with larger samples and multivariable analysis.

Secondly, the Internet survey methodology may have introduced bias, explaining for instance the unexpected high male to female ratio observed in this study. The Internet survey was a real limitation in France in 2008, with lower Internet penetration in the female population explaining the overestimation of males in the diabetic population, as in 2010 this bias was less important with a rate of $59 \%$ of males much closer to the normal rate of $54 \%$ in type 2 diabetes. ${ }^{37}$ Also, due to the self-report nature of the current study, no verification of diagnoses, treatment, fasting glucose, $\mathrm{HbA}_{1 \mathrm{c}}$ level or disease complications was made.

Third, cross-sectional data provide a one-time snapshot of the relationships between study variables. They can suggest directions for further research, but definite claims cannot be made regarding causal relationships among domains (eg, earlier insulin initiation and quality of life or adherence). However, the relevance of this data is strong because of the comparable methodology; 3 years of data can be pulled and a larger sample size is achievable, the patients reported are looking at many different measures using validated scales (the MMAS and SF-12v2) and even more are looking at treatment satisfaction, all these dimensions that can only have been caught from the patient perspective.

\section{Conclusion}

With these limitations in mind, the current study contributes to the growing literature documenting the burden and health effects associated with insulin treatment. There may be a rationale for prescribing insulin earlier than what is done with the current treatment paradigm. ${ }^{5}$ Recently, the effect of introducing insulin early in the course of the disease was reported in the ORIGIN (Outcome Reduction with Initial Glargine Intervention insuliN glargine therapy) study. ${ }^{48}$ The effect on prevention of mortality was neutral in this study. 
However, early insulin initiation was shown to be safe, leading to a modest increase in body weight and in the rate of severe hypoglycemia, and was reassuring concerning the risk of cancer. There was a reduction in diabetes incidence in individuals having only prediabetes at entry to the study. Thus, given the potential impact of prescribing insulin earlier, the current paper provides important information regarding the experience of insulin users in France. Finally, the main finding of our study was an unexpected improvement in adherence among insulin-treated patients, and the absence of a deleterious effect on quality of life in patients with no complications. This may represent an argument to fight against psychological insulin resistance.

\section{Acknowledgments}

The authors are grateful for their fruitful discussions with Florence Morin Riou, a consultant from Kantar Health, and Patricia Perlès, an employee of Sanofi, who participated in the writing of the manuscript.

\section{Disclosure}

The NHWS is conducted by Kantar Health. Sanofi France purchased access to the NHWS dataset and funded the analysis for this project, including fees for the author. GR has received honoraria for giving lectures in symposia organized by Abbott, Astra Zeneca, Bayer Diagnostics, Dexcom, Lifescan, Lilly, Menarini, Merck-Serono, MSD, Novartis, Novo-Nordisk, Roche Diagnostics, and Sanofi-Aventis. He has also served on the advisory boards of Abbott, Bayer Diagnostics, Lifescan, Roche Diagnostics, and Sanofi-Aventis, and has received a grant from Lifescan for evaluating an educational tool used in functional insulin therapy. The authors have no other conflicts of interest in this work.

\section{References}

1. Hu FB. Globalization of diabetes. The role of diet, lifestyle, and genes. Diabetes Care. 2011;34:1249-1257.

2. Holman RR, Paul SK, Bethel MA, Matthews DR, Neil HA. 10-year follow-up of intensive glucose control in type 2 diabetes. $N$ Engl J Med. 2008;359:1577-1589.

3. Inzucchi SE, Bergenstal RM, Buse JB, et al. Management of hyperglycaemia in type 2 diabetes: a patient-centered approach. Position statement of the American Diabetes Association (ADA) and the European Association for the Study of Diabetes (EASD). Diabetologia. 2012;55:1577-1596.

4. Ismail-Beigi F, Moghissi E, Tiktin M, Hirsch IB, Inzucchi SE, Genuth S. Individualizing glycemic targets in type 2 diabetes mellitus: implications of recent clinical trials. Ann Intern Med. 2011;154:554-559.

5. Hsu WC. Consequences of delaying progression to optimal therapy in patients with type 2 diabetes not achieving glycemic goals. South Med J. 2009; $102: 67-76$.

6. Turner RC, Holman RR. Insulin rather than glucose homeostasis in the pathophysiology of diabetes. Lancet. 1976;1:1272-1274.
7. Le Roith D, Fonseca V, Vinik A. Metabolic memory in diabetes - focus on insulin. Diabetes Metab Res Rev. 2005;21:85-90.

8. Leslie CA, Satin-Rapaport W, Matheson D, et al. Psychological insulin resistance: a missed diagnosis. Diabetes Spectr. 1994;7:52-57.

9. Phillips LS, Branch WT Jr, Cook CB, et al. Clinical inertia. Ann Intern Med. 2001;135:825-834.

10. Balkau B, Boué S, Avignon A et al. Type 2 diabetes treatment intensification in general practice in France in 2008-2009: The DIAttitude study. Diabetes Metab. 2012;38: S29-S35.

11. Brown JB, Nichols GA. Slow response to loss of glycemic control in type 2 diabetes mellitus. Am J Manag Care. 2003;9:213-217.

12. El-kebbi IM, Ziemer DC, Gallina DL, Dunbar V, Phillips LS. Diabetes in urban African-Americans. Identification of barriers to provider adherence to management protocols. Diabetes Care. 1999;22: 1617-1620.

13. Harris SB, Kapor J, Lank CN, Willan AR, Houston T. Clinical inertia in patients with T2DM requiring insulin in family practice. Can Fam Physician. 2010;56:e418-e424.

14. Shah BR, Hux JE, Laupacis A, Zinman B, Van Walraven C. Clinical inertia in response to inadequate glycemic control do specialists differ from primary care physicians? Diabetes Care. 2005;28:600-606.

15. Wallace TM, Matthews DR. Poor glycaemic control in type 2 diabetes: a conspiracy of disease, suboptimal therapy and attitude. QJM. 2000;93:369-374.

16. Peyrot M, Rubin RR, Lauritzen T, et al; International DAWN Advisory Panel. Resistance to insulin therapy among patients and providers: results of the cross-national Diabetes Attitudes, Wishes, and Needs (DAWN) study. Diabetes Care. 2005;28:2673-2679.

17. Peyrot M, Rubin RR, Khuntic K, et al. Addressing barriers to initiation of insulin in patients with type 2 diabetes. Prim Care Diabetes. 2010;4 Suppl 1:11-18.

18. Phillips P. Type 2 diabetes - failure, blame and guilt in the adoption of insulin therapy. Rev Diabetic Stud. 2005;2:35-39.

19. Woudenberg YJ, Lucas C, Latour C, Scholte op Reimer WJ. Acceptance of insulin therapy: a long shot? Psychological insulin resistance in primary care. Diabet Med. 2012;29:796-802.

20. Campbell RK. Recommendations for improving adherence to type 2 diabetes mellitus therapy-focus on optimizing insulin-based therapy. $\mathrm{Am}$ J Manag Care. 2012;18(3):S55-61.

21. Peyrot M, Barnett AH, Meneghini LF, Schumm-Draeger PM. Factors associated with injection omission/non-adherence in the Global Attitudes of Patients and Physicians in Insulin Therapy study. Diabetes Obes Metab. 2012;9999:9999.

22. Peyrot M, Rubin RR, Kruger DF, Travis LB. Correlates of insulin injection omission. Diabetes Care. 2010;33:240-245.

23. Schmittdiel JA, Uratsu CS, Karter AJ, et al. Why don't diabetes patients achieve recommended risk factor targets? Poor adherence versus lack of treatment intensification. J Gen Intern Med. 2008;23:588-594.

24. Ware JE, Kosinski M, Turner-Bowker DM, et al. How to Score Version 2 of the SF-12 Health Survey (with a supplement documenting version 1). Lincoln, RI: Quality Metric; 2002.

25. Morisky DE, Green LW, Levine DM. Concurrent and predictive validity of a self-reported measure of medication adherence. Med Care. 1986;24:67-74.

26. Desai M, Clark A. Autoimmune diabetes in adults: lessons from the UKPDS. Diabet Med. 2008; Suppl 2:30-34.

27. Wamala S, Merlo J, Bostrom G, Hogstedt C, Agren G. Socioeconomic disadvantage and primary non-adherence with medication in Sweden. Int J Qual Health Care. 2007;19:134-140.

28. Jotkowitz AB, Rabinowitz G, Raskin Segal A, Weitzman R, Epstein L, Porath A. Do patients with diabetes and low socioeconomic status receive less care and have worse outcomes? A national study. Am J Med. 2006;119:665-669.

29. Duru OK, Bilik D, McEwen LN, et al. Primary language, income and the intensification of anti-glycemic medications in managed care: the (TRIAD) study. J Gen Intern Med. 2011;26:505-511. 
30. Lawrence DB, Ragucci KR, Long LB, Parris BS, Helfer LA. Relationship of oral antihyperglycemic (sulfonylurea or metformin) medication adherence and hemoglobin A1c goal attainment for HMO patients enrolled in a Diabetes Disease Management Program. J Manag Care Pharm. 2006;12:466-471.

31. Rozenfeld Y, Hunt JS, Plauschinat C, Wong KS. Oral antidiabetic medication adherence and glycemic control in managed care. Am J Manag Care. 2008;14:71-75.

32. Parris ES, Lawrence DB, Mohn LA, Long LB. Adherence to statin therapy and LDL cholesterol goal attainment by patients with diabetes and dyslipidemia. Diabetes Care. 2005;28:595-599.

33. Bramley TJ, Gerbino PP, Nightengale BS, Frech-Tamas F. Relationship of blood pressure control to adherence with antihypertensive monotherapy in 13 managed care organizations. J Manag Care Pharm. 2006;12:239-245.

34. Briesacher BA, Andrade SE, Fouayzi H, Chan KA. Comparison of drug adherence rates among patients with seven different medical conditions. Pharmacotherapy. 2008;28:437-443.

35. Lee R, Taira DA. Adherence to oral hypoglycemic agents in Hawaii. Prev Chronic Dis. 2005;2:A09.

36. Cooke CE, Lee HY, Tong YP, Haines ST. Persistence with injectable antidiabetic agents in members with type 2 diabetes in a commercial managed care organization. Curr Med Res Opin. 2010;26:231-238.

37. Tiv M, Viel JF, Mauny F, et al. Medication adherence in type 2 diabetes: the ENTRED Study 2007, a French population-based study. PLoS One. 2012; 7:e32412.

38. Ahmed AT, Karter AJ, Liu J. Alcohol consumption is inversely associated with adherence to diabetes self-care behaviours. Diabet Med. 2006;23:795-802.
39. Reach G. Obedience and motivation as mechanisms for adherence to medication: a study in obese type 2 diabetic patients. Patient Prefer Adherence. 2011;5:523-531.

40. Jiménez-García R, Esteban-Hernández J, Hernández-Barrera V, et al. Clustering of unhealthy lifestyle behaviors is associated with nonadherence to clinical preventive recommendations among adults with diabetes. J Diabetes Complications. 2011;25:107-113.

41. Chiu CJ, Wray LA. Factors predicting glycemic control in middle-aged and older adults with type 2 diabetes. Prev Chronic Dis. 2010;7:A08.

42. Toljamo M, Hentinen M. Adherence to self-care and glycaemic control among people with insulin-dependent diabetes mellitus. J Adv Nurs. 2001;34:780-786.

43. Karter AJ, Ferrara A, Darbinian JA, Ackerson LM, Selby JV. Self-monitoring of blood glucose: language and financial barriers in a managed care population with diabetes. Diabetes Care. 2000;23: $477-483$.

44. Grégoire JP, Moisan J, Guibert R, et al. Determinants of discontinuation of new courses of antihypertensive medications. J Clin Epidemiol. 2002;55:728-735

45. Babiker MA. Compliance with penicillin prophylaxis by children with impaired splenic function. Trop Geogr Med. 1986;38:119-122.

46. Partridge AH. Non-adherence to endocrine therapy for breast cancer. Ann Oncol. 2006;17:183-184.

47. Nakar S, Yitzhaki G, Rosenberg R, Vinker S. Transition to insulin in type 2 diabetes: family physicians' misconception of patients' fears contributes to existing barriers. J Diabetes Complications. 2007;21:220-226.

48. ORIGIN Trial Investigators; Gerstein HC, Bosch J, Dagenais GR, et al. Basal insulin and cardiovascular and other outcomes in dysglycemia N Engl J Med. 2012;367:319-328.
Patient Preference and Adherence

\section{Publish your work in this journal}

Patient Preference and Adherence is an international, peer-reviewed, open access journal focusing on the growing importance of patient preference and adherence throughout the therapeutic continuum. Patient satisfaction, acceptability, quality of life, compliance, persistence and their role in developing new therapeutic modalities and compounds to

\section{Dovepress}

optimize clinical outcomes for existing disease states are major areas of interest. This journal has been accepted for indexing on PubMed Central The manuscript management system is completely online and includes a very quick and fair peer-review system. Visit http://www.dovepress.com/ testimonials.php to read real quotes from published authors. 